\title{
Coverage and Ergodic Rate in $K$-Tier Downlink Heterogeneous Cellular Networks
}

\author{
Harpreet S. Dhillon, Radha Krishna Ganti, François Baccelli and Jeffrey G. Andrews
}

\begin{abstract}
Cellular networks are becoming increasingly heterogeneous due to the co-deployment of many disparate infrastructure elements, including micro, pico and femtocells, and distributed antennas. This introduces new challenges in the modeling, analysis, and design of these networks. While gridbased models have been quite popular in modeling classical macrocell networks, they are both analytically intractable and have limited applicability to heterogeneous cellular networks (HCNs). We propose a flexible, accurate and tractable model for a general downlink HCN consisting of $K$ tiers of randomly located BSs, where each tier may differ in terms of average transmit power, supported data rate, and BS density. Assuming 1) a mobile connects to the strongest $\mathrm{BS}$, and 2) received power is subject to Rayleigh fading and path loss, we derive expressions for the coverage probability, ergodic rate and the average rate conditioned on the mobile being in coverage as the functions of target Signal-to-Interference-Ratio (SIR).
\end{abstract}

\section{INTRODUCTION}

Cellular networks are becoming increasingly complex due to the co-deployment of various classes of BSs both in space and frequency [1]. High power macro-cell BSs are already being joined by operator managed pico-cells [2], [3], compact form-factor distributed antennas [4]-[6], and low-power userinstalled femtocells [7], [8]. As a result, the traditional macrocell based networks have evolved into HCNs, where BSs may differ by a few orders of magnitude in terms of their transmit power and the density in which they are deployed. For example, it is easy to imagine a LTE network consisting of macro, pico and femtocells, with transmit powers of $50 \mathrm{~W}$, $2 \mathrm{~W}$ and $200 \mathrm{~mW}$, respectively, where perhaps 10 pico-cells and 100 femtocells exist in a high power macro-cell, sharing the same licensed spectrum. Naturally, the experience of mobile users in terms of coverage, rate and reliability would be quite different in these networks as compared to the familiar macrocell based networks. This increasing heterogeneity complicates the problem of modeling and analysis of cellular networks, which is known to be fairly hard even in the case of classical homogeneous cellular networks.

The research into modeling and analysis of cellular networks has predominantly been influenced by two main approaches. For academic research, very simple models are typically used to maintain tractability. The most popular analytical model even to the present day is the Wyner model, which assumes channel gain from all (typically 1 or 2 ) interfering

H. S. Dhillon, R. K. Ganti and J. G. Andrews are with WNCG, the University of Texas at Austin, USA. Email: dhillon@utexas.edu, rganti@austin.utexas.edu, jandrews@ece.utexas.edu. F. Baccelli is with Ecole Normale Superieure (ENS) and INRIA in Paris, France. Email: Francois.Baccelli@ens.fr.
BSs are equal and thus interference power is constant over the entire cell [9]. This model suffers from two main drawbacks: 1) it does not distinguish between the cell-edge and the cellinterior, and 2) in most cases, it does not have a notion of outage since the signal-to-interference-ratio (SIR) is fixed and deterministic [10]. On the other hand, for design and development (e.g., in industry) complex system-level simulations with large number of system parameters are generally used. The most popular and widely accepted simulation model is the two-dimensional hexagonal grid model. While it is flexible and allows to study the effect of various system parameters, it is generally not tractable [11], [12]. Moreover, in the context of network heterogeneity and randomness in the BS locations, its accuracy is questionable.

A third and relatively new method of studying cellular networks is to allow locations of the BSs to be drawn from a stochastic process [13]-[16] and then use stochastic geometry techniques to obtain tractable framework [17]. It was developed for macro-cell based cellular networks in [17] where the BS locations were modeled by a homogeneous Poisson Point Process (PPP). Perhaps surprisingly, it was shown that the resulting model is about as accurate as the grid model even though the actual macro-cell BSs are centrally placed and have no apparent randomness in their locations. In this paper, we extend this model to a $\mathrm{HCN}$ where it is likely even more sensible due to the presence of heterogeneous infrastructure elements such as femtocells. More precisely, the model consists of $K$ independent tiers of PPP distributed BSs, where each tier may differ in terms of the average transmit power, the supported data rate, and the BS density. Assuming 1) a typical mobile user connects to the strongest BS, and 2) Rayleigh fading, we derive exact expressions for the coverage probability, ergodic rate and the average rate conditioned on the mobile being in coverage.

This paper extends the results of [18], where the coverage probability and the average rate conditioned on the mobile being in coverage were obtained by assuming a target SIR greater than $0 \mathrm{~dB}$ for each tier. In this paper, we relax this condition and derive these results for the general target SIR. This also enables us to obtain the ergodic rate which was not possible under the assumptions of [18].

\section{SySTEM MODEL}

\section{A. Heterogeneous Cellular Network Model}

We model a $\mathrm{HCN}$ as a $K$-tier cellular network where each tier models the BSs of a particular type, such as those of femtocells or pico-cells. The BSs across tiers may differ in 


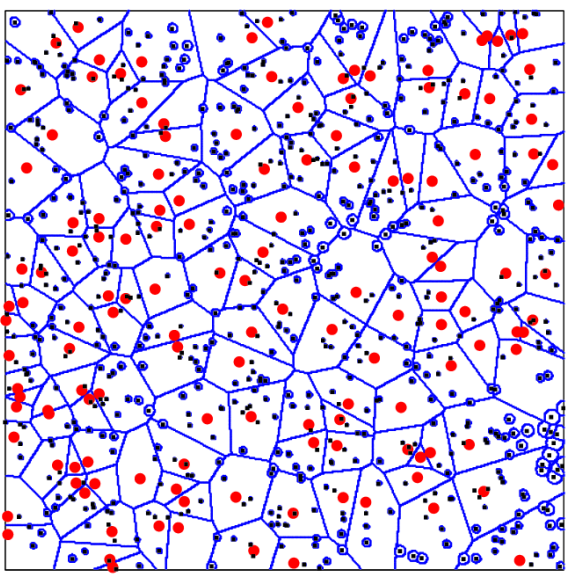

Fig. 1. Coverage regions in a two-tier network as per the model used in this paper. Both macro (large circles) and femto (small dark squares) BSs are distributed as independent PPPs with $P_{1}=1000 P_{2}$ and $\lambda_{2}=5 \lambda_{1}$.

terms of the transmit power, the supported data rate and their spatial density. We assume that the BSs in the $i$-th tier are spatially distributed as a PPP $\Phi_{i}$ of density $\lambda_{i}$, transmit at power $P_{i}$, and have a SIR target of $\beta_{i}$. More precisely a mobile can reliably communicate with a BS $x$ in the $i$-th tier only if its downlink SIR with respect to that BS is greater than $\beta_{i}$. The mobiles are also modeled by an independent PPP $\Phi_{m}$ of density $\lambda_{m}$. Without loss of generality, we assume that the typical mobile user is located at the origin. The fading (power) between a BS located at point $x$ and the typical mobile is denoted by $h_{x}$ and is assumed to be i.i.d exponential (Rayleigh fading). The standard path loss function is given by $l(x)=\|x\|^{-\alpha}$, where $\alpha>2$ is the path loss exponent. Hence, the received power at a typical mobile user from a BS located at point $x_{i}$ (belonging to $i^{\text {th }}$ tier) is $P_{i} h_{x_{i}}\left\|x_{i}\right\|^{-\alpha}$, where $h_{x_{i}} \sim \exp (1)$. The resulting SIR expression assuming the user connects to this BS is:

$$
\operatorname{SIR}\left(x_{i}\right)=\frac{P_{i} h_{x_{i}}\left\|x_{i}\right\|^{-\alpha}}{\sum_{j=1}^{K} \sum_{x \in \Phi_{j} \backslash x_{i}} P_{j} h_{x}\|x\|^{-\alpha}},
$$

Since interference typically dominates noise in all cellular networks of even modest density, we neglect thermal noise in this work, which further improves tractability. We will comment more on this assumption in the Numerical Results Section. We assume that each mobile user connects to its strongest BS instantaneously, i.e., the BS that offers the highest received SIR. Mathematically the typical node at the origin is in coverage if:

$$
\max _{x \in \Phi_{i}} \operatorname{SIR}(x)>\beta_{i}
$$

for some $1 \leq i \leq K$. The model considers a single frequency band and assumes that all BSs are transmitting continuously in all time slots at constant power, although if some fraction $f$ of time slots were not used (at random), then the resulting density of interfering BSs would simply be $(1-f) \lambda$ and the analysis could be extended.

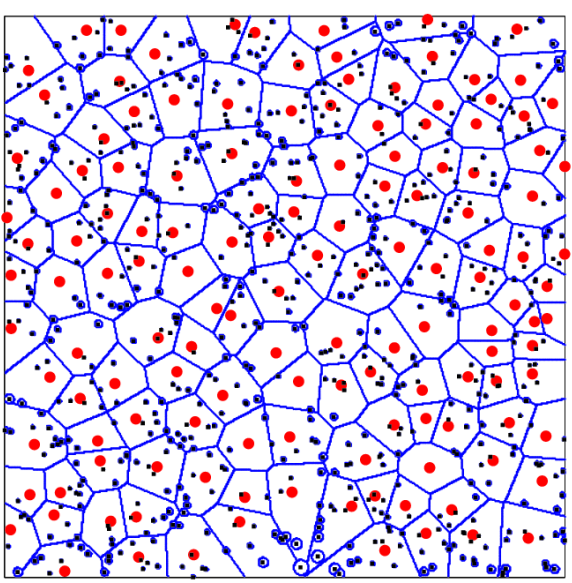

Fig. 2. Coverage regions in a two-tier network where Macro (tier-1) BS locations (large circles) correspond to actual $4 \mathrm{G}$ deployment in an urban area. Femto BSs (small dark squares) are distributed as a PPP $\left(P_{1}=1000 P_{2}\right.$ and $\left.\lambda_{2}=5 \lambda_{1}\right)$.

\section{B. Coverage Regions}

The illustrative HCN coverage regions under no-fading assumption can be visually plotted in two steps, resulting in Figs. 1-3. First, we randomly place $K$ different types of BSs on a 2 -D plane according to the aforementioned independent PPPs. Second, the space is fully tessellated following the maximum SIR (equivalently maximum power) connectivity model in the absence of fading. Due to the differences in the transmit powers over the tiers, the coverage plots do not correspond to a standard Voronoi tessellation [19]. Instead, they closely resemble a circular Dirichlet tessellation, also called a multiplicatively weighted Voronoi diagram [20]. The coverage regions for a two-tier network - for example comprising macro and femtocells - are depicted in Figs. 1 and 2 for two cases: 1) the macro-cell BSs are distributed according to PPP (our model), and 2) the macro-cell BSs correspond to an actual $4 \mathrm{G}$ deployment over a relatively flat urban region. The femtocells are distributed according to an independent PPP in both cases. Qualitatively, the coverage regions are quite similar in the two cases. The close-up view of coverage regions with an additional pico-tier is shown in Fig. 3. Apart from the apparent heterogeneity, this coverage footprint highlights the particularly important role of smaller cells where macrocell coverage is poor.

\section{Coverage Probability}

We first provide the coverage probability of a typical user which is required to obtain the average rate in the next section.

Theorem 1. The coverage probability of a typical mobile user is

$$
\begin{gathered}
\mathrm{P}_{\mathrm{c}}\left(\left\{\lambda_{i}\right\},\left\{\beta_{i}\right\},\left\{P_{i}\right\}\right)=\frac{2 \pi \Gamma\left(1+\frac{2}{\alpha}\right)}{\alpha} \sum_{m=1}^{K} \lambda_{m}\left(P_{m} \delta_{m}\right)^{1+\frac{2}{\alpha}} \\
\cdot \int_{-\infty}^{\infty} \int_{0}^{\infty} \frac{\mathcal{L}_{I}^{y}(j 2 \pi \omega)}{y^{1+2 / \alpha}} \frac{e^{j 2 \pi \omega y\left(1-\delta_{m}^{-1}\right)}-e^{j 2 \pi \omega y\left(\kappa^{-1}-\delta_{m}^{-1}\right)}}{j 2 \pi \omega} \mathrm{d} y \mathrm{~d} \omega .
\end{gathered}
$$




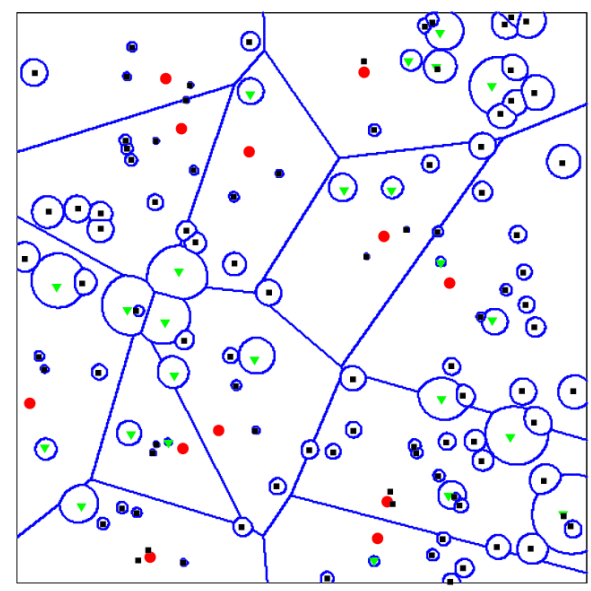

Fig. 3. Close-up view of coverage regions in a three-tier network. All the tiers, i.e., tier-1 macro (large circles), tier-2 pico (light triangles) and tier-3 femto (small dark squares), are modeled as independent PPPs. $P_{1}=100 P_{2}=$ $1000 P_{3}, \lambda_{3}=4 \lambda_{2}=8 \lambda_{1}$.

In the above expression $\delta_{i}=\frac{1+\beta_{i}}{\beta_{i}}, \kappa=\max \delta_{i}$ and

$$
\mathcal{L}_{I}^{u}(s)=e^{-\pi \sum_{m=1}^{K} \lambda_{m}\left(s P_{m}\right)^{\frac{2}{\alpha}} \Gamma\left(1+\frac{2}{\alpha}\right)\left[\Gamma\left(1-\frac{2}{\alpha}\right)+2 / \alpha \Gamma\left(-\frac{2}{\alpha}, \frac{s u}{\delta_{m}}\right)\right]}
$$

Proof: Since the BSs are distributed as a stationary process, we can consider a typical mobile at the origin. The coverage probability of a typical mobile user at the origin is

$$
1-\mathbb{E}\left[\prod_{i=1}^{K} 1-\mathbf{1}\left(\frac{M_{i}}{I-M_{i}}>\beta_{i}\right)\right]
$$

where

$$
M_{i}=P_{i} \max _{x \in \Phi_{i}}\left\{h_{x}\|x\|^{-\alpha}\right\},
$$

and $I$ is the total aggregate interference at the origin given by

$$
I=\sum_{i=1}^{K} \sum_{x \in \Phi_{i}} P_{i} h_{x}\|x\|^{-\alpha} .
$$

By basic algebra, the coverage probability equals

$$
\mathrm{P}_{\mathrm{c}}\left(\left\{\lambda_{i}\right\},\left\{\beta_{i}\right\},\left\{P_{i}\right\}\right)=\mathbb{P}\left(\max _{i}\left(\delta_{i} M_{i}\right)>I\right) .
$$

Let

$$
\mathcal{L}_{I}^{u}(s)=\mathbb{E}\left[e^{-s I} \mathbf{1}\left(\max _{i}\left(\delta_{i} M_{i}\right)<u\right)\right] .
$$

It can be reduced to (2) using [21, Theorem 1]. Differentiating $\mathcal{L}_{I}^{u}(s)$, we obtain

$$
\left.\frac{\partial \mathcal{L}_{I}^{y}(s)}{\partial y}\right|_{u}=\frac{\mathcal{L}_{I}^{u}(s) 2 \pi \Gamma\left(1+\frac{2}{\alpha}\right)}{\alpha u^{1+2 / \alpha}} \sum_{m=1}^{K} \lambda_{m}\left(P_{m} \delta_{m}\right)^{1+\frac{2}{\alpha}} e^{-\frac{s u}{\delta_{m}}} .
$$

Let $f(x, y)$ denote the joint probability density of $I$ and $\max \left(\delta_{i} M_{i}\right)$. The coverage probability equals

$$
\mathrm{P}_{\mathrm{c}}=\int_{0}^{\infty} \int_{0}^{\infty} f(x, y) \mathbf{1}\left(y>x>\frac{y}{\max \left(\delta_{i}\right)}\right) \mathrm{d} x \mathrm{~d} y .
$$

Let $\kappa=\max \left(\delta_{i}\right)$. Using Parsevals theorem ${ }^{1}$, we obtain

$$
\mathrm{P}_{\mathrm{c}}=\int_{0}^{\infty} \int_{-\infty}^{\infty} \hat{f}(\omega, y) \frac{e^{j 2 \pi \omega y}-e^{j 2 \pi \omega y / \kappa}}{j 2 \pi \omega} \mathrm{d} \omega \mathrm{d} y,
$$

where $\hat{f}(\omega, y)$ denotes the Fourier transform of $f(x, y)$ with respect to the $x$ variable. We also have

$$
\frac{\partial \mathcal{L}_{I}^{u}(s)}{\partial u}=\int_{x=0}^{\infty} f(x, y) e^{-j 2 \pi \omega x} \mathrm{~d} x=\hat{f}(\omega, y) .
$$

Interchanging the integrals in (4) and using the above equation, we obtain the result.

The above theorem provides coverage probability for all values of $\beta_{i}>0$; however the coverage probability requires evaluation of complex integrals. An assumption that greatly simplifies the analysis is that $\beta_{i}>1$, i.e., the SIR threshold for connectivity to any tier is greater than $0 \mathrm{~dB}$. The following Lemma shows that under this assumption, a mobile can connect to at most one BS in the entire network. Although some users in commercial cellular networks indeed have operating SIR below $0 \mathrm{~dB}$, they are in a distinct minority (cell edge users) and in the Numerical Results section we show that the results obtained with $\beta_{i}>1$ assumption hold very accurately to about $-4 \mathrm{~dB}$, which covers cell edge users as well.

Lemma 1. Given positive real numbers $\left\{a_{1}, a_{2} \ldots a_{n}\right\}$, which correspond to the received power from each $B S$ at the typical mobile user and defining $b_{i}=\frac{a_{i}}{\sum_{j \neq i} a_{j}}$, which corresponds to the SIR of the $i^{\text {th }} B S$, at most $m b_{i}$ 's can be greater than $1 / m$ for any positive integer $m$.

Proof: See [18].

The model is applicable both to $\mathrm{CDMA}^{2}$ and OFDMAbased cellular networks. The next theorem specializes Theorem 1 , to $\beta_{i}>1$.

Theorem 2. When $\beta_{i}>1$, the coverage probability for a typical mobile user in open access is

$\mathrm{P}_{\mathrm{c}}\left(\left\{\lambda_{i}\right\},\left\{\beta_{i}\right\},\left\{P_{i}\right\}\right)=\frac{\pi}{C(\alpha)} \frac{\sum_{i=1}^{K} \lambda_{i} P_{i}^{2 / \alpha} \beta_{i}^{-2 / \alpha}}{\sum_{i=1}^{K} \lambda_{i} P_{i}^{2 / \alpha}}, \quad \beta_{i}>1$,

where $C(\alpha)=2 \pi^{2} \alpha^{-1} \csc (2 \pi / \alpha)$.

Proof: We now provide the intuition behind the proof. For a detailed proof, please refer to [18]. The coverage probability is

$$
\mathrm{P}_{\mathrm{c}}=1-\mathbb{E} \prod_{m=1}^{K}\left(\prod_{x \in \Phi_{m}}\left(1-\mathbf{1}\left(\operatorname{SIR}(x)>\beta_{k}\right)\right)\right) .
$$

When $\beta_{i}>1$, from Theorem 1 , atmost one BS can connect to the mobile at the origin. Hence for any $a \geq 2$, and $x_{1}, \ldots, x_{a}$, we have

$$
\prod_{b=1}^{a} \mathbf{1}\left(\operatorname{SIR}\left(x_{b}\right)>\beta_{k}\right)=0 \text {. }
$$

\footnotetext{
${ }^{1}$ This requires verifying that the integrand is integrable, which we do not provide in this paper. The technique for verifying the integrability is common and can be found in [22].

${ }^{2}$ For CDMA networks, although the received SIR is generally much smaller than 1 , the post-despreading SIR, which is what determines coverage/outage, is often greater than or at least close to 1 , so our model and Lemma 1 are still reasonable if the interference term is divided by a spreading factor $M$.
} 
So we have,

$$
\mathrm{P}_{\mathrm{c}}=\mathbb{E} \sum_{m=1}^{K} \sum_{x \in \Phi_{m}} \mathbf{1}\left(\operatorname{SIR}(x)>\beta_{k}\right) .
$$

Moving the expectation inside the summation, and using Cambell-Mecke theorem [23], and the fact that interference is $\alpha$-stable, we obtain the result.

\section{Average Rate}

In this section, we focus on the study of the average communication rate achievable by a typical mobile user in the $K$ tier HCN. Throughout this study, we assume that the capacity achieving codes are used and hence the Shannon's capacity formula, $R=\log \left(1+\max _{x \in \cup_{i} \Phi_{i}} \operatorname{SIR}(x)\right)$ bps/Hz, is applicable. It is worth noting here that the $\mathrm{P}_{\mathrm{c}}$ expression derived in Theorem 1 completely characterizes the complementary cumulative distribution function (ccdf) of $\max _{x \in \cup_{i}} \operatorname{SIR}(x)$ and hence holds a key to the derivation of the ergodic rate. We now derive the Ergodic rate $\mathbb{E}[R]$ using the coverage probability result derived in Theorem 1.

\section{A. Ergodic Rate}

Theorem 3. The ergodic rate achievable by a typical mobile in a K-tier $\mathrm{HCN}$ is given by:

$$
\mathbb{E}[R]=\int_{0}^{\infty} \mathrm{P}_{\mathrm{c}}\left\{\left\{\lambda_{i}\right\}, 2^{y}-1,\left\{P_{i}\right\}\right\} \mathrm{d} y .
$$

$\mathrm{P}_{\mathrm{c}}\left\{\left\{\lambda_{i}\right\}, 2^{y}-1,\left\{P_{i}\right\}\right\}$ is the complex integral expression for coverage probability derived in Theorem 1 by fixing $\beta_{i}=$ $2^{y}-1 \forall i$.

Proof: Let $X$ be the random variable denoting $\max _{x \in \cup_{i} \Phi_{i}} \operatorname{SIR}(x)$ and $R$ be the random variable denoting in$x \in \cup_{i} \Phi_{i}$

stantaneous rate $\log (1+X)$ in bps/Hz. Since $R$ is a positive random variable, its expected value can be evaluated as:

$$
\begin{aligned}
E[R] & =\int_{0}^{\infty} \mathbb{P}[R \geq y] \mathrm{d} y=\int_{0}^{\infty} \mathbb{P}\left[X \geq 2^{y}-1\right] \mathrm{d} y \\
& =\int_{0}^{\infty} \mathrm{P}_{\mathrm{c}}\left\{\left\{\lambda_{i}\right\}, 2^{y}-1,\left\{P_{i}\right\}\right\} \mathrm{d} y,
\end{aligned}
$$

which completes the proof.

\section{B. Average Rate in Coverage}

Often, the service providers are interested in knowing the average rate they can provide to the users that are under coverage. Motivated by this reason, we now obtain the average rate $\bar{R}$ achievable by a typical mobile user conditioned on it being in coverage. For tractability, we assume $\beta_{i}>1$ for all tiers in the rest of this section. The main result for the average rate in coverage is given in Theorem 4.
Theorem 4. For $\beta_{i}>1$, the average rate achievable by a typical mobile when it is in coverage is

$$
\bar{R}=\log \left(1+\beta_{\text {min }}\right)+\frac{\sum_{i=1}^{K} \lambda_{i} P_{i}^{2 / \alpha} \mathcal{A}\left(\alpha, \beta_{i}, \beta_{\text {min }}\right)}{\sum_{i=1}^{K} \lambda_{i} P_{i}^{2 / \alpha} \beta_{i}^{-2 / \alpha}},
$$

where

$$
\mathcal{A}\left(\alpha, \beta_{i}, \beta_{\text {min }}\right)=\int_{\beta_{\text {min }}}^{\infty} \frac{\max \left(\beta_{i}, x\right)^{-2 / \alpha}}{1+x} \mathrm{~d} x,
$$

and $\beta_{\min }=\min \left\{\beta_{1}, \beta_{2}, \ldots, \beta_{K}\right\}$.

Proof: Let $\mathbf{C}\left(\left\{\beta_{i}\right\}\right)$ denote the event that the typical mobile user is in coverage, i.e., the typical user at the origin is able to connect to some BS. Hence, this event equals $\bigcup_{i=1}^{K} \bigcup_{x \in \Phi_{i}}\left(\operatorname{SIR}(x)>\beta_{i}\right)$. Since $\mathbf{C}\left(\left\{\beta_{i}\right\}\right)$ is the event that the typical mobile use is in coverage, it follows that the coverage probability provided in Theorem 2 is $\mathrm{P}_{\mathrm{c}}\left(\left\{\lambda_{i}\right\},\left\{\beta_{i}\right\},\left\{P_{i}\right\}\right)=\mathbb{P}\left(\mathbf{C}\left(\left\{\beta_{i}\right\}\right)\right)$. We are interested in the average rate when the typical user is in coverage, which can be mathematically expressed in terms of conditional expectation as:

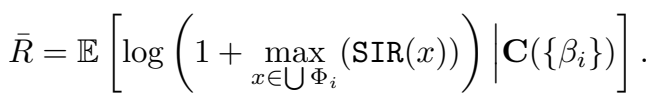

Denote the random variable $\max _{x \in \bigcup} \Phi_{i}(\operatorname{SIR}(x))$ by $X$. We first derive the conditional complementary cumulative density function (CCDF) of $X$. Using Bayes' Theorem,

$$
\mathbb{P}\left(X>T \mid \mathbf{C}\left(\left\{\beta_{i}\right\}\right)\right)=\frac{\mathbb{P}\left(X>T, \mathbf{C}\left(\left\{\beta_{i}\right\}\right)\right)}{\mathbb{P}\left(\mathbf{C}\left(\left\{\beta_{i}\right\}\right)\right)},
$$

Since, $X>T$ denotes the event that the maximum SIR that the typical user sees is larger than $T$, it is also equal to $\bigcup_{i=1}^{K} \bigcup_{x \in \Phi_{i}}(\operatorname{SIR}(x)>T)$. Hence,

$$
\mathbb{P}\left(X>T, \mathbf{C}\left(\left\{\beta_{i}\right\}\right)\right)=\mathbb{P}\left(\mathbf{C}(\{T\}), \mathbf{C}\left(\left\{\beta_{i}\right\}\right)\right)
$$

Using the basic properties of sets, it follows that $\mathbf{C}(\{T\}) \cap$ $\mathbf{C}\left(\left\{\beta_{i}\right\}\right)=\mathbf{C}\left(\left\{\max \left(T, \beta_{i}\right)\right\}\right)$. Hence,

$$
\mathbb{P}\left(X>T \mid \mathbf{C}\left(\left\{\beta_{i}\right\}\right)\right)=\frac{\mathbb{P}\left(\mathbf{C}\left(\left\{\max \left(T, \beta_{i}\right)\right\}\right)\right)}{\mathbb{P}\left(\mathbf{C}\left(\left\{\beta_{i}\right\}\right)\right)} .
$$

Using Theorem 2, and denoting $\min \left\{\beta_{1}, \beta_{2}, \ldots, \beta_{K}\right\}$ by $\beta_{\text {min }}$, we obtain

$\mathbb{P}\left(X>T \mid \mathbf{C}\left(\left\{\beta_{i}\right\}\right)\right)=\left\{\begin{array}{c}\frac{\sum_{i=1}^{K} \lambda_{i} P_{i}^{2 / \alpha} \max \left(\beta_{i}, T\right)^{-2 / \alpha}}{\sum_{i=1}^{K} \lambda_{i} P_{i}^{2 / \alpha} \beta_{i}^{-2 / \alpha}} ; T>\beta_{\text {min }} \\ 1 ; \quad \text { otherwise. }\end{array}\right.$

Using the conditional CCDF, $\bar{R}$ can be evaluated as follows:

$$
\begin{aligned}
\bar{R} & =\int_{0}^{\infty} \log (1+x) f_{X}\left(x \mid \mathbf{C}\left(\left\{\beta_{i}\right\}\right)\right) \mathrm{d} x, \\
& =\int_{x=0}^{\infty} \int_{y=0}^{x} \frac{1}{1+y} f_{X}\left(x \mid \mathbf{C}\left(\left\{\beta_{i}\right\}\right)\right) \mathrm{d} y \mathrm{~d} x, \\
& \stackrel{(a)}{=} \int_{y=0}^{\infty}\left(\int_{x=y}^{\infty} f_{X}\left(x \mid \mathbf{C}\left(\left\{\beta_{i}\right\}\right)\right) \mathrm{d} x\right) \frac{1}{1+y} \mathrm{~d} y, \\
& =\int_{0}^{\infty} \frac{\mathbb{P}\left(X>y \mid \mathbf{C}\left(\left\{\beta_{i}\right\}\right)\right)}{1+y} \mathrm{~d} y,
\end{aligned}
$$


where $(a)$ follows from changing the order of integration. Now we substitute (12) in (13) to obtain

$$
\begin{aligned}
\bar{R} & =\int_{0}^{\beta_{\min }} \frac{1}{1+y} d y+\frac{\sum_{i=1}^{K} \lambda_{i} P_{i}^{2 / \alpha} \int_{\beta_{\min }}^{\infty} \frac{\max \left(\beta_{i}, x\right)^{-2 / \alpha}}{1+x} \mathrm{~d} x}{\sum_{i=1}^{K} \lambda_{i} P_{i}^{2 / \alpha} \beta_{i}^{-2 / \alpha}}, \\
& =\log \left(1+\beta_{\min }\right)+\frac{\sum_{i=1}^{K} \lambda_{i} P_{i}^{2 / \alpha} \int_{\beta_{\min }}^{\infty} \frac{\max \left(\beta_{i}, x\right)^{-2 / \alpha}}{1+x} \mathrm{~d} x}{\sum_{i=1}^{K} \lambda_{i} P_{i}^{2 / \alpha} \beta_{i}^{-2 / \alpha}} .
\end{aligned}
$$

This completes the proof.

Thus we observe that the average rate expression involves only a single integral which can be easily evaluated numerically.

Corollary 1. When all the tiers have the same SIR threshold $\beta>1$, the average rate achievable by a randomly chosen mobile that is in coverage in open access networks is

$$
\bar{R}=\log (1+\beta)+\beta^{2 / \alpha} \mathcal{A}(\alpha, \beta, \beta) .
$$

The above result shows that the average rate is independent of the density of BSs of each tier when the SIR thresholds are same for all the tiers. This is expected because the distribution of max SIR does not depend upon the density of BSs in this case (this follows from Theorem 2 by setting $\beta_{i}=\beta \forall i$ ).

\section{Closed Access}

So far, all the results pertain to an open access network, where a mobile is allowed to connect to any BS in the network. We now focus on the closed access case, also known as a closed subscriber group, where a mobile user is allowed to connect to only a subset of tiers and the rest of the tiers act purely as interferers. The motivation for closed access particularly applies to privately owned infrastructure, such as femtocells or perhaps custom picocells mounted on a company's roof to improve service to their staff. As in the previous case, we first state the result for coverage probability of a randomly located mobile in closed access in Lemma 2 (assuming $\beta_{i}>1$ for all tiers). Please refer to [18] for the proof.

Lemma 2. When a typical mobile user is allowed to connect to only a subset $\mathcal{B} \subset\{1,2, \ldots, K\}$, the coverage probability for the closed access scheme with $\beta_{i}>1$ is

$$
\mathrm{P}_{\mathrm{c}}\left(\left\{\lambda_{i}\right\},\left\{\beta_{i}\right\},\left\{P_{i}\right\}\right)=\frac{\pi}{C(\alpha)} \frac{\sum_{i \in \mathcal{B}} \lambda_{i} P_{i}^{2 / \alpha} / \beta_{i}^{2 / \alpha}}{\sum_{i=1}^{K} \lambda_{i} P_{i}^{2 / \alpha}} .
$$

Now the average rate achievable by a randomly chosen mobile under closed access (assuming it is under coverage) can be expressed as

$\bar{R}_{c}=\mathbb{E}\left[\log \left(1+\max _{x \in \bigcup_{i \in \mathcal{B}} \Phi_{i}}(\operatorname{SIR}(x))\right) \mid \bigcup_{\substack{x \in \Phi_{i} \\ i \in \mathcal{B}}}\left(\operatorname{SIR}(x)>\beta_{i}\right)\right]$.

Following the same steps as in proof of Theorem 4, we arrive at the following Proposition.

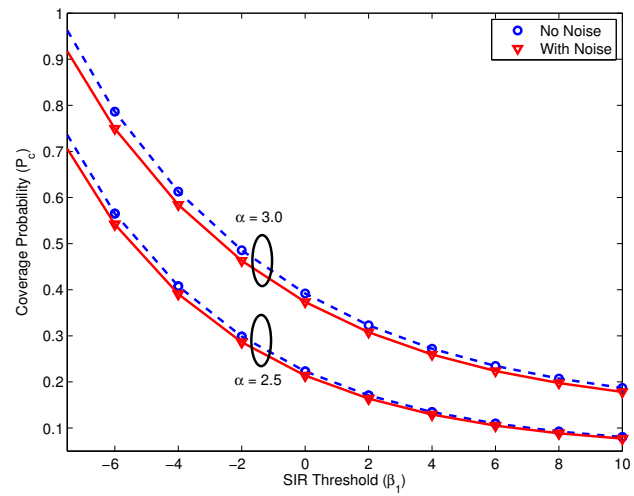

Fig. 4. Coverage probability in a two-tier $\mathrm{HCN}$ with and without thermal noise $\left(K=2, P_{1}=25 P_{2}, \lambda_{2}=5 \lambda_{1}, \beta_{2}=1 \mathrm{~dB}, \mathrm{SNR}_{e d g e}=0 \mathrm{~dB}\right)$. The "with-noise" plot is generated from the coverage probability expression derived in [18, Theorem 1].

Proposition 1. Assuming a mobile user is allowed to connect to only a subset $\mathcal{B}$ of the $K$ tiers (with $\beta_{i}>1$ ), the average rate conditioned on the mobile being in coverage is

$$
\bar{R}_{c}=\log \left(1+\beta_{\text {min }}\right)+\frac{\sum_{i \in \mathcal{B}} \lambda_{i} P_{i}^{2 / \alpha} \mathcal{A}\left(\alpha, \beta_{i}, \beta_{\text {min }}\right)}{\sum_{i \in \mathcal{B}} \lambda_{i} P_{i}^{2 / \alpha} \beta_{i}^{-2 / \alpha}},
$$

where

$$
\mathcal{A}\left(\alpha, \beta_{i}, \beta_{\text {min }}\right)=\int_{\beta_{\text {min }}}^{\infty} \frac{\max \left(\beta_{i}, x\right)^{-2 / \alpha}}{1+x} \mathrm{~d} x,
$$

and $\beta_{\text {min }}=\min \left\{\beta_{i}\right\}$, where $i \in \mathcal{B}$.

Corollary 2. Assuming the threshold of each tier is the same and equal to $\beta$, the average rate achievable by a randomly chosen mobile in coverage under closed access is

$$
\bar{R}_{c}=\log (1+\beta)+\beta^{2 / \alpha} \mathcal{A}(\alpha, \beta, \beta) .
$$

From Corollaries 1 and 2, we observe that the average rate $(\bar{R})$ of the mobile while it is in coverage is not affected by access control when the thresholds are the same for all tiers. However, please recall that since the coverage probability is lower in case of closed access, it will lead to a lower ergodic rate as compared to an open access network.

\section{Numerical Results}

\section{A. Validating No-Noise Assumption}

To validate the no-noise assumption, we study the effect of thermal noise on the coverage probability in a typical twotier network consisting of macro-cells overlaid with pico-cells. We again assume that the mobile user connects to the BS with the strongest downlink signal $(\max (\mathrm{SINR})$ or equivalently $\max (\mathrm{SIR})$ connectivity model). To set the reference noise power, we use the following notion of cell-edge users in this example. Defining the distance of the the nearest macro BS to the typical mobile user to be $d$ and the underlying random variable to be $D$, the mobile user is said to be on the cell edge if $\mathbb{P}(D \leq d) \geq P_{\text {edge }}$, where $P_{\text {edge }}$ is set to 0.9 for this illustration. For PPP of intensity $\lambda_{1}, \mathbb{P}(D \leq d)=1-\exp \left(\lambda_{1} \pi d^{2}\right)$, 


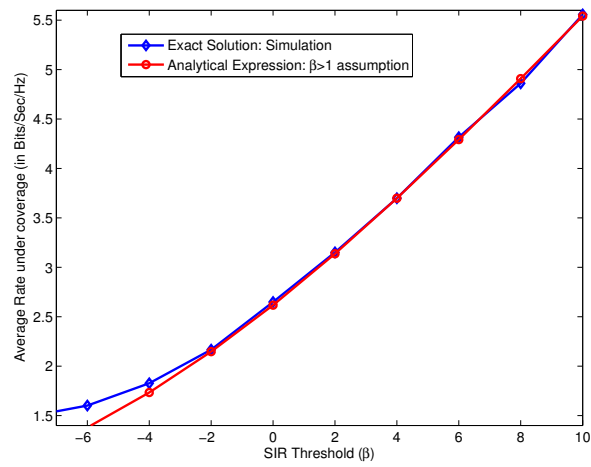

Fig. 5. Average rate while mobile is in coverage ( $K=2, \alpha=3, P_{1}=$ $1000 P_{2}, \lambda_{2}=2 \lambda_{1}, \beta_{1}=\beta_{2}=\beta$, no noise, open access).

giving $d \geq \sqrt{\frac{-\ln \left(1-P_{\text {edge }}\right)}{\pi \lambda_{1}}}$. For a desired edge-user SNR, say $\mathrm{SNR}_{\text {edge }}$, the noise power $\sigma^{2}$ can be approximated as $\sigma^{2} \approx \frac{P_{1} d_{\text {edge }}^{-\alpha}}{\text { SNR }_{\text {edge }}}$, where $d_{\text {edge }}=\sqrt{\frac{-\ln \left(1-P_{\text {edge }}\right)}{\pi \lambda_{1}}}$. Please note that we have inherently assumed the first tier to be the macro tier. Under this setup, we present the coverage probability for various values of $\alpha$ in Fig. 4. By comparing these results with the no-noise case, we note that the typical HCNs are interference limited and hence thermal noise has a very limited effect on coverage probability. This validates the no-noise assumption.

\section{B. Average Rate and $\beta>1$ Assumption}

The average rate under coverage $(\bar{R})$ for a two-tier HCN is plotted in Fig. 5. From the plot, we note that the analytical results obtained under $\beta>1$ assumption provide a reasonably tight bound even until $\beta=-4 \mathrm{~dB}$. Therefore, the simplified analytical results also cover the cell edge users having operating SIR below $0 \mathrm{~dB}$ (typically not lower than $-3 \mathrm{~dB}$ in commercial cellular networks).

\section{CONCLUSIONS}

We have developed a new tractable model for downlink HCNs consisting of $K$ tiers of randomly located BSs, where each tier may differ in terms of the average transmit power, target SIR and the supported data rate. Using this model, we have obtained exact expressions for the coverage probability, ergodic rate and the average rate conditioned on the mobile being in coverage. This model reinforces the usefulness of random spatial models in the analysis and research of cellular networks. This is a baseline tractable HCN model with possible future extensions being the inclusion of frequency reuse, power control and interference avoidance/ cancellation.

\section{REFERENCES}

[1] Qualcomm, "LTE advanced: heterogeneous networks," white paper, Jan. 2011.

[2] X. Wu, B. Murherjee, and D. Ghosal, "Hierarchical architectures in the third-generation cellular network," IEEE Trans. on Wireless Communications, vol. 11, no. 3, pp. 62 - 71, 2004.

[3] S. Kishore, L. Greenstein, H. Poor, and S. Schwartz, "Uplink user capacity in a CDMA macrocell with a hotspot microcell: exact and approximate analyses," IEEE Trans. on Wireless Communications, vol. 2, no. 2, pp. $364-374$, Mar. 2003.
[4] W. Rho and A. Paulraj, "Performance of the distributed antenna systems in a multicell environment," in Proc., IEEE Veh. Technology Conf., Apr. 2003, pp. 587-591.

[5] J. Zhang and J. G. Andrews, "Distributed antenna systems with randomness," IEEE Trans. on Wireless Communications, vol. 7, no. 9, pp. 3636-3646, Sep. 2008.

[6] W. Choi and J. G. Andrews, "Downlink performance and capacity of distributed antenna systems in a multicell environment," IEEE Trans. on Wireless Communications, pp. 69-73, Jan. 2007.

[7] Picochip, "The case for home base stations," white paper, Apr. 2007.

[8] V. Chandrasekhar, J. G. Andrews, and A. Gatherer, "Femtocell networks: A survey," IEEE Comm. Mag., vol. 46, no. 9, pp. 59-67, Sep. 2008.

[9] A. D. Wyner, "Shannon-theoretic approach to a Gaussian cellular multiple-access channel," IEEE Trans. on Info. Theory, vol. 40, no. 6 , pp. 1713-1727, Nov. 1994.

[10] J. Xu, J. Zhang, and J. G. Andrews, "On the accuracy of the Wyner model in cellular networks," submitted, 2010, available at: arxiv.org/abs/1009.5900.

[11] A. Ganz, C. M. Krishna, D. Tang, and Z. J. Haas, "On optimal design of multitier wireless cellular systems," IEEE Comm. Mag., vol. 35, no. 2, pp. 88-93, Feb. 1997.

[12] E. Ekici and C. Ersoy, "Multi-tier cellular network dimensioning," $A C M$ Wireless Networks, vol. 7, no. 4, pp. 401-411, Aug. 2001.

[13] P. Fleming, A. Stolyar, and B. Simon, "Closed-form expressions for other-cell interference in cellular CDMA," Report no. 116, Center for Computational Mathematics, University of Colorado, Denver, Dec. 1997.

[14] T. X. Brown, "Cellular performance bounds via shotgun cellular systems," IEEE Journal on Sel. Areas in Communications, vol. 18, no. 11, pp. $2443-55$, Nov. 2000 .

[15] F. Baccelli, M. Klein, M. Lebourges, and S. Zuyev, "Stochastic geometry and architecture of communication networks," J. Telecommunication Systems, 1997.

[16] F. Baccelli and S. Zuyev, "Stochastic geometry models of mobile communication networks," in Frontiers in queueing. Boca Raton, FL: CRC Press, 1997, pp. 227-243.

[17] J. G. Andrews, F. Baccelli, and R. K. Ganti, "A tractable approach to coverage and rate in cellular networks," IEEE Trans. on Communications, Submitted, Sept. 2010, available at: arxiv.org/abs/1009.0516.

[18] H. S. Dhillon, R. K. Ganti, F. Baccelli, and J. G. Andrews, "Modeling and analysis of K-tier downlink heterogeneous cellular networks," IEEE Journal on Sel. Areas in Communications, Submitted, Mar. 2011, available at: arxiv.org/abs/1103.2177.

[19] F. Aurenhammer, "Voronoi diagrams - A survey of a fundamental geometric data structure," ACM Computing Surveys, vol. 23, no. 3, pp. 345-405, Sep. 1991.

[20] P. Ash and E. Bolker, "Generalized Dirichlet tessellations," Geometriae Dedicata, vol. 20, no. 2, pp. 209-243, 1986.

[21] V. Nguyen and F. Baccelli, "A stochastic geometry model for the best signal quality in a wireless network," in Proc. IEEE Int. Symposium on Modeling and Optimization in Mobile, Ad Hoc and Wireless Networks (WiOpt), 2010, pp. 465-471.

[22] F. Baccelli, B. Blaszczyszyn, and P. Muhlethaler, "Stochastic analysis of spatial and opportunistic Aloha," IEEE Journal on Sel. Areas in Communications, vol. 27, pp. 1105-1119, 2009.

[23] D. Stoyan, W. Kendall, and J. Mecke, Stochastic Geometry and Its Applications, 2nd ed. John Wiley and Sons, 1996. 